The Cryosphere Discuss., 4, 1433-1448, 2010 www.the-cryosphere-discuss.net/4/1433/2010/ doi:10.5194/tcd-4-1433-2010

\title{
Interplay between linear, dissipative and permanently critical mechanical processes in Arctic sea ice
}

A. Chmel ${ }^{1}$, V. Smirnov ${ }^{2}$, and A. Panov ${ }^{2}$

${ }^{1}$ Ioffe Physico-Technical Institute, St.-Petersburg, Russia

${ }^{2}$ Arctic and Antarctic Research Institute, St.-Petersburg, Russia

Received: 8 June 2010 - Accepted: 20 July 2010 - Published: 25 August 2010

Correspondence to: A. Chmel (chmel@mail.ioffe.ru)

Published by Copernicus Publications on behalf of the European Geosciences Union.

Mechanical processes in Arctic sea ice

A. Chmel et al.

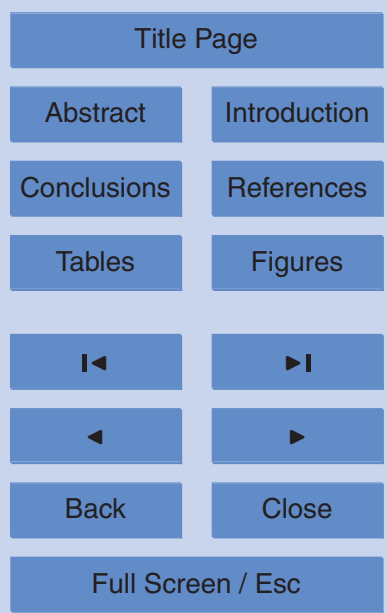

Printer-friendly Version

Interactive Discussion 


\section{Abstract}

Mechanical processes in the Arctic ice pack result in fragmented sea ice cover, the regular geometry of which could be described in main features in terms of the conventional mechanics. However, the size distribution of sea ice floes does not exhibit

5 the random (poissonian-like) statistics and follows the power law typical for self-similar (fractal) structures. The analysis of ice floe oscillations in the frequency range specific for cracking, shearing and stick-slip motion evidences the self-organized dynamics of sea ice fracturing, which manifests itself in scaling distributions of both the discrete energy discharges in fracture events and the recurrence times between that one. So determined space-time-energy self-similarity characterises the ice pack as the nonequilibrium, nonlinear thermodynamic system where the synergic relations are established through conventional long propagating wave/oscillations. The presented experimental data were collected at the Russian ice-research camp "North Pole 35" drifting on the Arctic ice pack in 2008.

\section{Introduction}

The Arctic sea ice cover (ASIC) is a complicated mechanical system, with components which could spontaneously decrease or increase dimensions in cycles of fragmentation and freezing. In addition, independent from mechanical perturbations, the ice floe geometry, as well as the floe size distribution, maintains the same relations, some of which could be explained in the framework of conventional mechanics of solids, while some other ones represent the nonlinear nature of the pack dynamics. The mechanical state of the sea ice cover is important to navigation and marine engineering in cold regions, as well as for climate change through the albedo variation. Therefore, the adequate description of the pack behaviour is necessary for estimating and forecasting important changes in the state of sea ice cover.

\section{Mechanical \\ processes in Arctic sea ice}
A. Chmel et al.

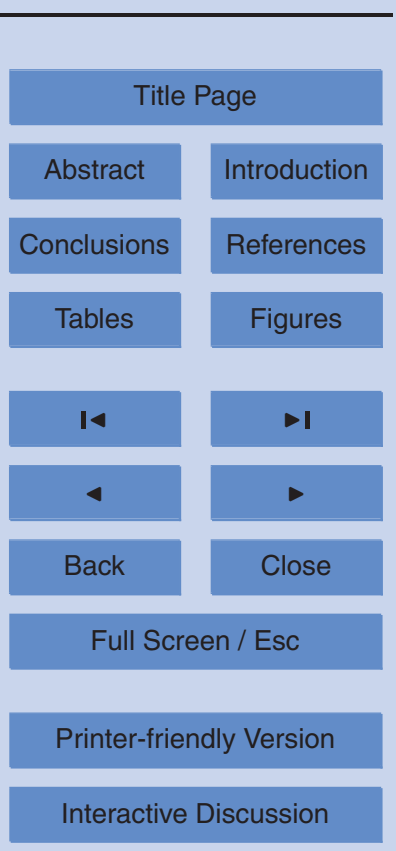


We present a brief overview of the main trends in the mechanical and physical behaviour of the ASIC by drawing attention to the interplay between, conditionally speaking, mechanical and thermodynamic features in the dynamics of sea ice. The main source of the experimental data was the database collected at the Russian sea ice 5 research camps "North Pole" in 2008 and other years.

\section{Geometry and size distribution}

The large-scale visualization of the ASIC, which became available from satellite systems in the early seventies, revealed a rectilinear lead pattern ranging from 1 to $10^{2} \mathrm{~km}$. The most impressive finding was the parallelogram-like structure of the pattern with 10 the constant angle $\sim 30^{\circ}$ between directions of intersecting leads (Fig. 1). However, it was revealed later that this regular structure is not a unique feature inherent in the ASIC; fracturing of this kind have a direct analogy in the rock mechanics where threedimensional strike slip faults occur as a result of the semi-brittle failure of horizontally stressed rock formations. Marco and Thompson (1977) explained the $\sim 30^{\circ}$ difference 15 in the orientation of leads on the basis of the Coulomb-Mohr law, which establishes conditions by which the medium deforms on account of shape changes without increasing stresses. The Mohr theory of failure suggests that the applied shear stress at failure, $\tau$, and the normal stress, $\sigma_{n}$, on the failure plane (or line in 2-D-case) are related by

$20 \tau=C+\sigma_{n} \tan \varphi$

where $C$ is the material cohesion strength; $\varphi$ is the angle of internal friction, $\varphi= \pm\left(\alpha-45^{\circ}\right)$; and $\alpha$ is the intersection angle. The resolution of Eq. (1), in relation to the intersection angle with parameters characteristic for sea ice, gives one $\alpha \approx 30^{\circ}$, that is the value observed in many satellite images.

$25 \quad$ Marco and Thompson (1977) pointed out also that the parallelogram-like pattern appears only at sufficiently rapid strain rates when the semi-brittle failure prevails.
4, 1433-1448, 2010

\section{Mechanical \\ processes in Arctic sea ice}

A. Chmel et al.

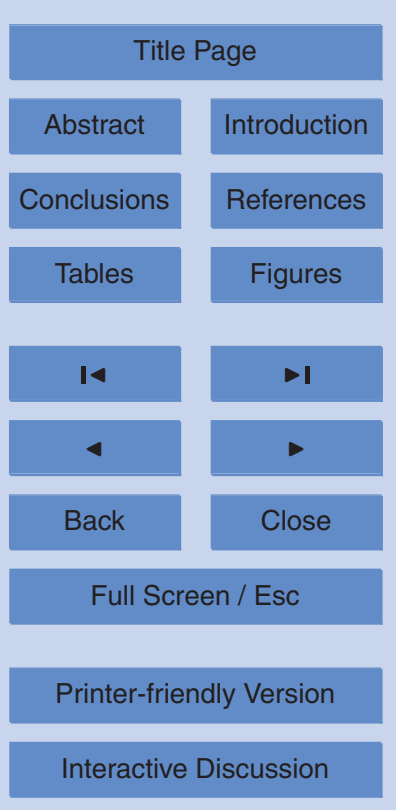

Interactive Discussion 
At relatively slow strain rates, the ductile behaviour takes place, which results in the formation of polygonal (diamond-like) or fully disordered structures due to cohesion flow at low yield stress.

Erlingsson (1988) drew attention to the fact that the Mohr theory of constant fric5 tion between sliding surfaces has no characteristic length, that is, it is scale invariant. Therefore, the angular relationship is valid over the whole area where the conditions of semi-brittle failure are satisfied. Thus, the self-similarity of the uniangular geometry of ice floes is the direct consequence of the Mohr theory.

However, further investigations clarified some features in the self-similar lead pattern, 10 which cannot be explained in the framework of conventional mechanics. Rothrock and Thorndike (1984) and Matushita (1985) studied the size distribution of sea ice floes and revealed that the number of floes no smaller than $\Sigma, N(>\Sigma)$, behaves as

$N(>\Sigma) \propto \Sigma^{-\alpha}$

where $\Sigma$ is the dimensional characteristic, such as area, $S$, or length, $L$. The power 15 law dependence means the dimensional self-similarity of the set of ice floes because Eq. (2) is equivalent to the scaling relation

$N(\lambda \Sigma)=\lambda^{-\alpha} N(\Sigma)$

where $\lambda$ is the constant (scaling factor). In general, the power law behaviour means the scale invariance of an object or process in space, time or energy (in dependence of the measured scaling parameter.)

Later, the power law size distribution was found for lead patterns of any kinds, that is both for ice floes of regular geometry, such as parallelogram-like and diamond-like floes, and for "smoothed" (oval) floes typical for frost-free seasons (Weiss and Marsan, 2004; Chmel et al., 2005, 2007). At the same time, the exponent $\beta$, in contrast to the parameter $\alpha$, is not a universal constant: its absolute value depends on relative amounts of "small" and "large" floes in the total $N$. This is seen in Fig. 2 where the $N(>S)$ versus $S$ dependences are constructed for summer and winter periods.

\section{Mechanical \\ processes in Arctic sea ice}

A. Chmel et al.

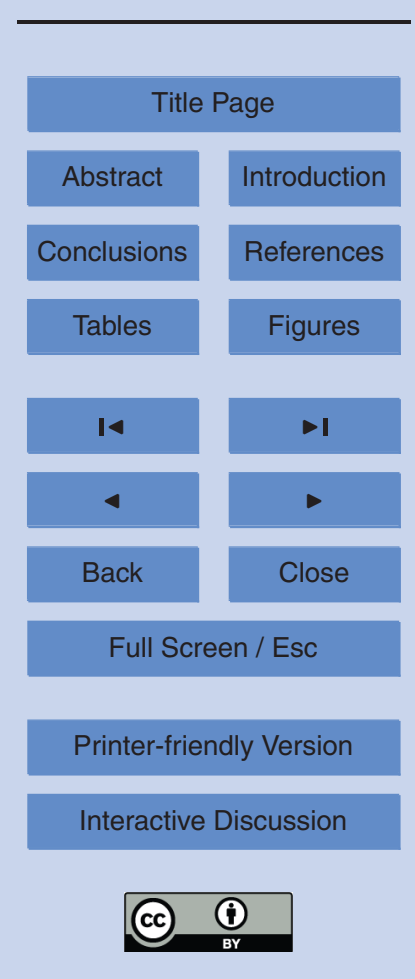


The slope of the summer log-linear dependence is significantly higher than the winter one in accordance with the prevalence of smaller floes in the size distribution measured during the frost-free season.

The size distribution (2) cannot be derived from the considerations based only on 5 conventional mechanics because the scaling behaviour of fracturing sea ice is the thermodynamic phenomenon that occurs in open, non-equilibrium systems referred as SOC-systems (SOC = self-organized criticality), in which scale-invariant distributions of their space, energy and time parameters exist permanently.

The criticality in this context implies the presence of long-range interactions between 10 system's components having no stable structural links between each other. In other words, the radius of correlated behaviour in SOC systems is much larger that the range of direct interactions between components.

In the case of dynamic, mechanical SOC system controlled by a non-regular external energy source, the multi-scale interactions adjust themselves spontaneously to 15 conserve the current critical state through events of localized failure which cause the stress/strain redistributions.

Thus, the scaling properties of a quasi-static ensemble of ice floes is a fingerprint of the critical dynamics in the ASIC, the main trends which can be revealed and assessed from the monitoring of the energy discharge in fracture events occurring in drifting ice.

\section{Waves and oscillations}

The fractal pattern of the sea ice cover implies the existence of a mechanism that would actually realize the persistent approaching of this mechanical system to its stable, scaling attractor. Apparently, this mechanism should be based on conventional physical processes capable in providing and maintaining long-range interactions between the system's components. In the ASIC, the fracturing consists of cracking, shearing and stick-slip events occurring over vast areas in which dimensions are determined by characteristic dimensions of ice pack fragments. These processes are detectable by

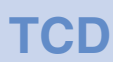

$4,1433-1448,2010$

\section{Mechanical \\ processes in Arctic sea ice}
A. Chmel et al.

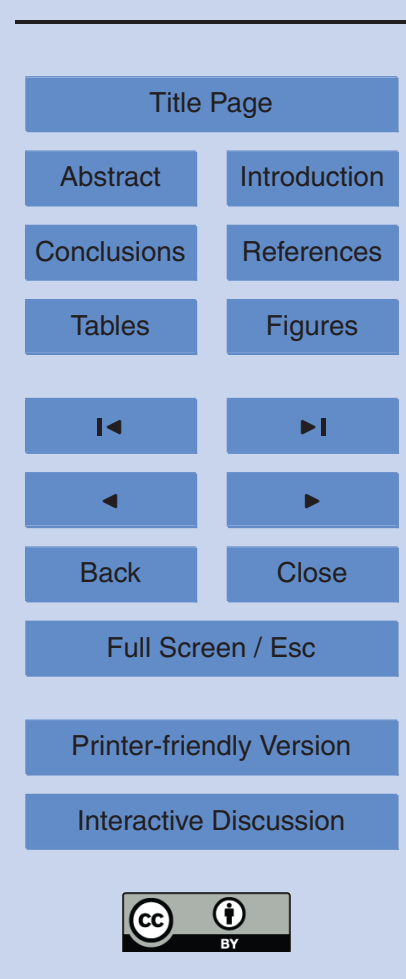


instruments sensitive to stresses and oscillations, which are commonly used in seismologic studies. The elastic waves emitted from local ice failures fall in the frequency range of around $1 \mathrm{~Hz}$. Figure 3 shows an example of the stress discharge in the drifting sea ice recorded simultaneously with two instruments, the stress sensor and the 5 seismic tiltmeter. The stress discharge of about $40 \mathrm{kPa}$ was followed by a short-term, sharp increase of the amplitude of oscillations with further frequency change. Such discharges are observable during the breakages of the ice continuity; the value of compression $(\sigma)$ is determined by Hooke's law. These phenomena are well described in the framework of conventional mechanics but the analysis of the amplitude and time 10 intervals between fracture events allows one to reveal some features of self-organizing typical for non-equilibrium critical systems.

In January, 2008, a continuous train of elastic waves that passed through an ice floe drifted in the Arctic region, shown in Fig. 1, was detected by a horizontal tiltmeter at the camp North Pole 35. In order to exclude low-frequency motions related with the swell forcing and gravimetric waves, the spectrum was filtered by a low-frequency cut-off equal to $0.5 \mathrm{~Hz}$. In addition, only relatively strong tilts that exceeded the instrumental accuracy at least two times were taken into account. So filtered and discriminated series are depicted in Fig. 4. The distribution of signal amplitudes, $A$, is equivalent to the energy distribution in detected stress discharges, since the primary tiltmeter response is formed by the instrument's pendulum, in which small deviations are proportional to the energy in exciting waves.

The energy distribution $N_{E}(>E)$ found from the recorded series is shown in Fig. 5 (here $N_{E}$ is the number of events, the energy release which is not smaller than $E$ ). The log-linear dependence means that the function $N_{\mathrm{E}}(>E)$ follows the power law

$25 \quad N_{\mathrm{E}}(>E) \propto E^{-b}$

Hence, the energy distribution of detected waves is self-similar in the sense of definition (Eq. 3).

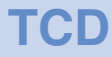

$4,1433-1448,2010$

\section{Mechanical \\ processes in Arctic sea ice}

A. Chmel et al.

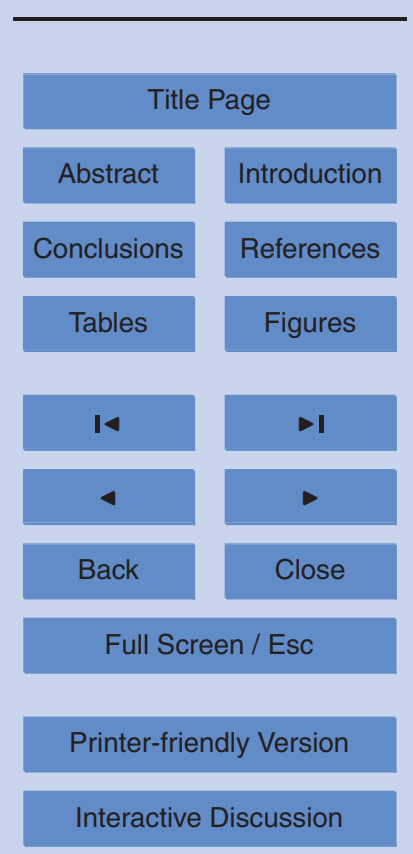




\section{Time series}

The energy series, such as shown in Fig. 4, allows one to determine the intervals between sufficiently strong signals and to construct the distribution function of the recurrence time between fracture events. Figure 6 shows the obtained distribution function,

$5 N_{\mathrm{t}}(>t) ; N_{\mathrm{t}}$ is the number of successive signals separated by the time interval exceeding $t$. One can see that the function $N_{\mathrm{t}}(>t)$ also exhibits the power law dependence:

$N_{\mathrm{t}}(>t) \propto t^{-\delta}$

which points out the time invariance of the fracture process in the interval of $\sim 10^{3} \mathrm{~s}$ determined by the range of the log-linear portions of the $N_{t}(>t)$ plot.

The time invariance is a representation of the long-term correlation between detected events. This "memory effect" is, in fact, a cooperative response of the system as a whole on the behaviour of its individual components. The highly variable contact interactions (stick-slips, impacts, etc.) play part of a dynamical structural link between mobile fragments of the sea ice cover.

\section{Discussion}

So, we presented some examples of self-organized processes in fracturing sea ice which manifest themselves in spatial, temporal and energy self-similarity of discrete events. Space and time self-organizing is specific for dynamic systems with nonlinearities of a different nature, in particular, with nonlinearities caused by specific interactions in non-equilibrium system. Changeability of interactions in multi-component, statistical systems promotes dynamic and structural fluctuations in their behaviour. In equilibrium systems, the fluctuations obey the law of large numbers; accordingly, the correlation lengths remain small both in space and time. In non-equilibrium, nonlinear systems, the random behaviour of components could spontaneously transform to a cooperative behaviour with the emerging of, so called, dissipative structures. The nucleation and evolution of dissipative structures are controlled by the order parameters of which certain values comply with the self-organized space-time trajectory of the system.

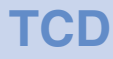

$4,1433-1448,2010$

\section{Mechanical \\ processes in Arctic sea ice}

A. Chmel et al.

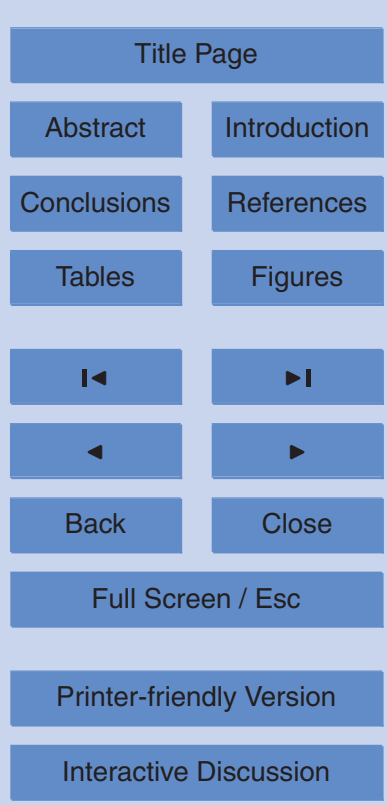


The latter point is of great importance in classifying the nature of a given nonlinear system. The tuning parameter is an attribute of Newtonian mechanics, which implies the deterministic character of the system's evolution. The Arctic sea ice cover is the open, non-equilibrium system, in which the phenomenon of self-organizing through 5 the dissipative mechanism can take place only under certain order parameters. In our case, one could see that at certain deformation rates some spatially correlated formations (parallelogram-like leads) occur in the ASIC; their scale of correlation covers, at least, three orders of magnitude. These dissipative structures reproduce themselves every time when the external forcing, such as wind forcing, provides the conditions of 10 semi-brittle failure.

In non-equilibrium systems, which in addition to the nonlinearity, possess a great number of metastable states (their numbers grow exponentially with the increase in the amount of system's components) the state of permanent criticality, SOC, that was mentioned above could appear. The SOC system's attractor is the boundary state 15 (between ordered - self-similar - structure and chaos), which is maintained by the external energy flow passing through the system (Obukhov, 1990). As distinct from dissipative structures, in which the appearance is conditioned by the pre-determined values of order parameters, the SOC state is not specified by any exact combination of tuning parameters; it does not depend on initial conditions and appears in a wide range of system's parameters. Its particular feature is the power law decay of correlations (in contrast to the exponential decay that takes place in dissipative systems). The data presented in this work demonstrate that the ASIC exhibits properties of both dissipative structures and SOC-systems.

At the same time, all the interactions that exist both in linear and nonlinear (selforganizing) systems are realized through conventional physical processes of establishing/breaking stable and metastable structural/dynamic links. To achieve the selforganization, these physical processes should exhibit a long-range spatial correlation; in the case of SOC systems, the time correlation (memory) is also necessary.

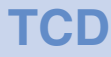

$4,1433-1448,2010$

\section{Mechanical \\ processes in Arctic sea ice}

A. Chmel et al.

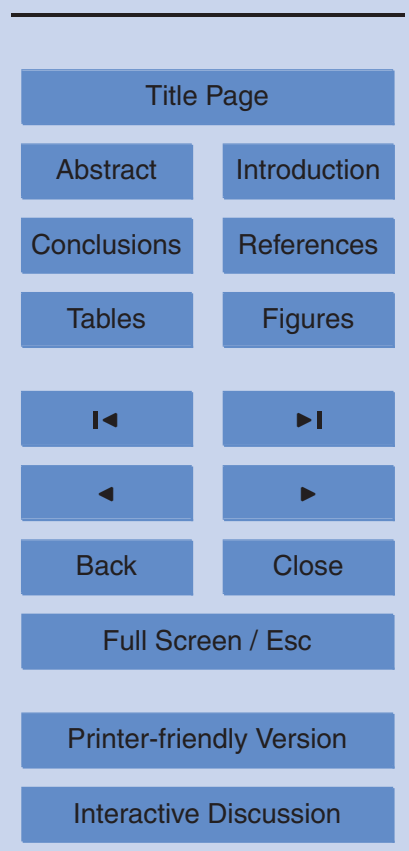


When considering an eventual mechanism of self-organizing, one should bear in mind that a substantial role in the ASIC behaviour is played by oscillation/wave processes. In the linear medium the oscillations are always chaotic, while in the nonlinear medium one could expect the emerging of cooperative oscillations whose response on the external forcing is not proportional to the actual action. Such the processes redistribute efficiently the energy between system's components, in particular, through parametric interactions. It is known that the oscillation motions in ice pack could involve ice floes separated by open cracks (Smirnov, 1996); these points out a possibility of cooperative phenomena in perturbing sea ice, including correlated in space and 10 time processes of deformation and fracture. In the above example (Fig. 6) the time correlation in the fracturing cycle reaches a few hours. Apparently, an individual ice floe incapable of storing the information about its prehistory, while the cooperative response of the environment on contact interactions depends on preceding events due to the stress/energy redistribution, which governs the virtual space-time trajectory of 15 the ice pack to its thermodynamically profitable attractor.

\section{Conclusions}

The processes of fracturing in drifting ice possess some features that cannot be explained completely in the framework of Newtonian mechanics. The conventional approach implies the linearity between the external forcing and the system's response. In general, this is not the case in drifting sea ice. Field experiments carried out on the ice pack demonstrate the space-time-energy invariance (fractality) of the fracture process. Statistical distributions of this kind are inherent to non-equilibrium physical processes that drive the critical phenomena. Therefore, the sea ice dynamics is, to a certain extent, the problem of the statistical physics of non-equilibrium critical systems.

The fractal behaviour represented by the power law distributions is of thermodynamic origin but the relevant synergic space-time effects are realized through the classical processes of energy exchange. With regards to the specific physical mechanism that

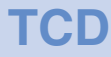

$4,1433-1448,2010$

\section{Mechanical \\ processes in Arctic sea ice}

A. Chmel et al.

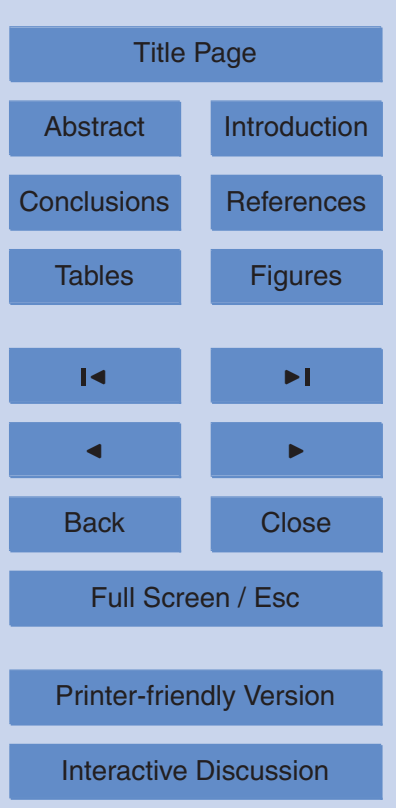

Interactive Discussion 
maintains the dynamic connectedness in the pack, the interactions between ice floes or between pack and air/ocean environment trigger intensive wave/oscillation processes including nonlinear ones, which perform the effective energy redistribution over vast areas, thus retaining the correlated fracturing of pack's components.

\section{References}

Chmel, A., Smirnov, V. N., and Astakhov, A. P.: The fractality of sea-ice drift dynamics as revealed from the "North Pole 32" monitoring, J. Stat. Mech., P02002, 1-11, 2005.

Chmel, A., Smirnov, V. N., and Panov, L. V.: Scaling aspects of the sea-ice-drift dynamics and pack fracture, Ocean Sci., 3, 291-298, doi:10.5194/os-3-291-2007, 2007.

10 Erlingsson, B.: Two-dimensional deformation patterns in sea ice, J. Glaciol., 34, 301-308, 1988.

Marco, J. R. and Thompson, R. E.: Rectilinear leads and internal motions in the ice pack of the Western Arctic Ocean, J. Geophys. Res., 82, 979-987, 1977.

Obukhov, S. P.: Self-organized criticality: Goldstone modes and their interactions, Phys. Rev. 15 Lett., 65, 1395-1398, 1990.

Rothrock, D. A. and Thorndike, A. S.: Measuring the sea-ice floe size distribution, J. Geophys. Res., 89, 6477-6486, 1984.

Smirnov, V. N.: Dynamic processes in sea ice, Gidrometeoizdat, St. Petersburg, Russia, 1996 (in Russian).

Weiss. J. and Marsan, D.: Scale properties of sea ice deformation and fracturing, C. R. Physique, 735-751, 2004.

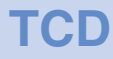

$4,1433-1448,2010$

\section{Mechanical \\ processes in Arctic sea ice}

A. Chmel et al.

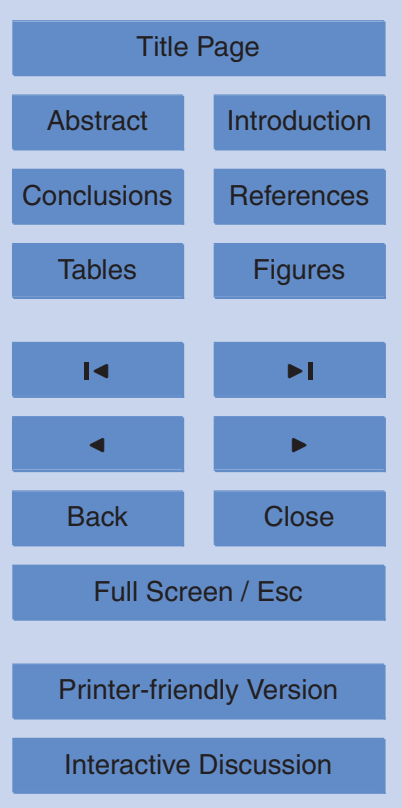




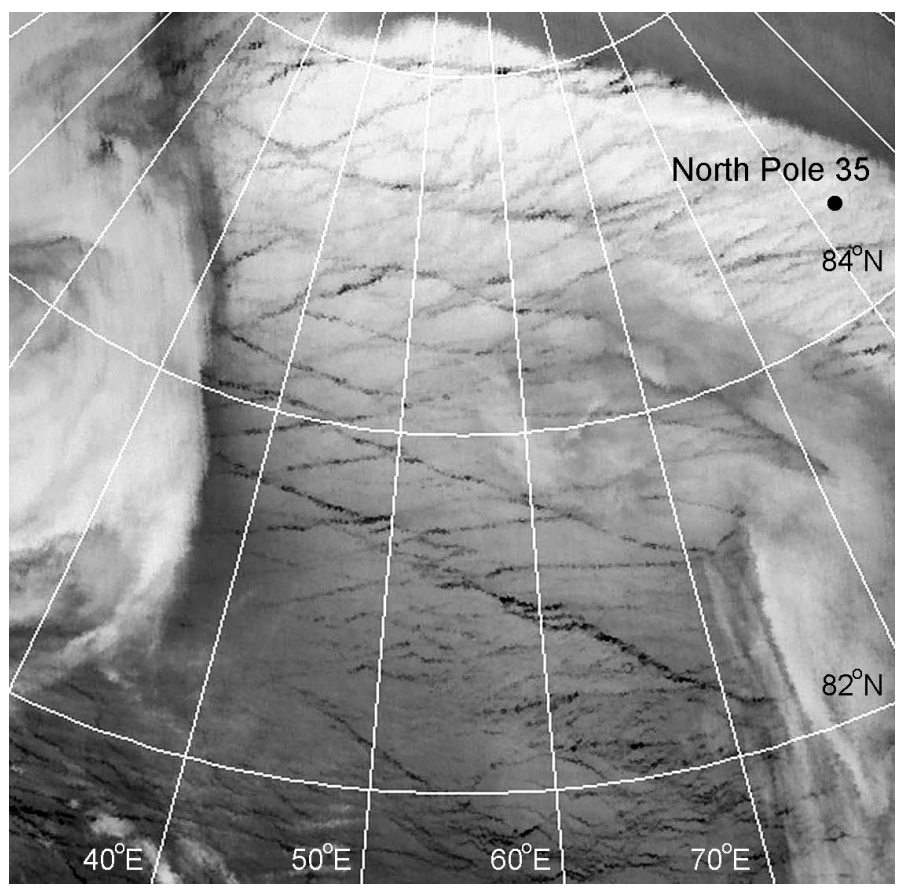

Fig. 1. The parallelogram-like crack-and-lead pattern in the ASIC in the region of activity of the research camp North Pole 35. The NOAA satellite image of 3 January 2008.

\section{TCD}

$4,1433-1448,2010$

\section{Mechanical processes in Arctic sea ice}

A. Chmel et al.

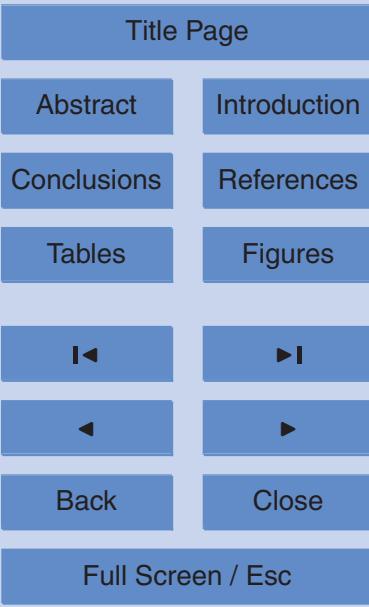

Printer-friendly Version

Interactive Discussion 


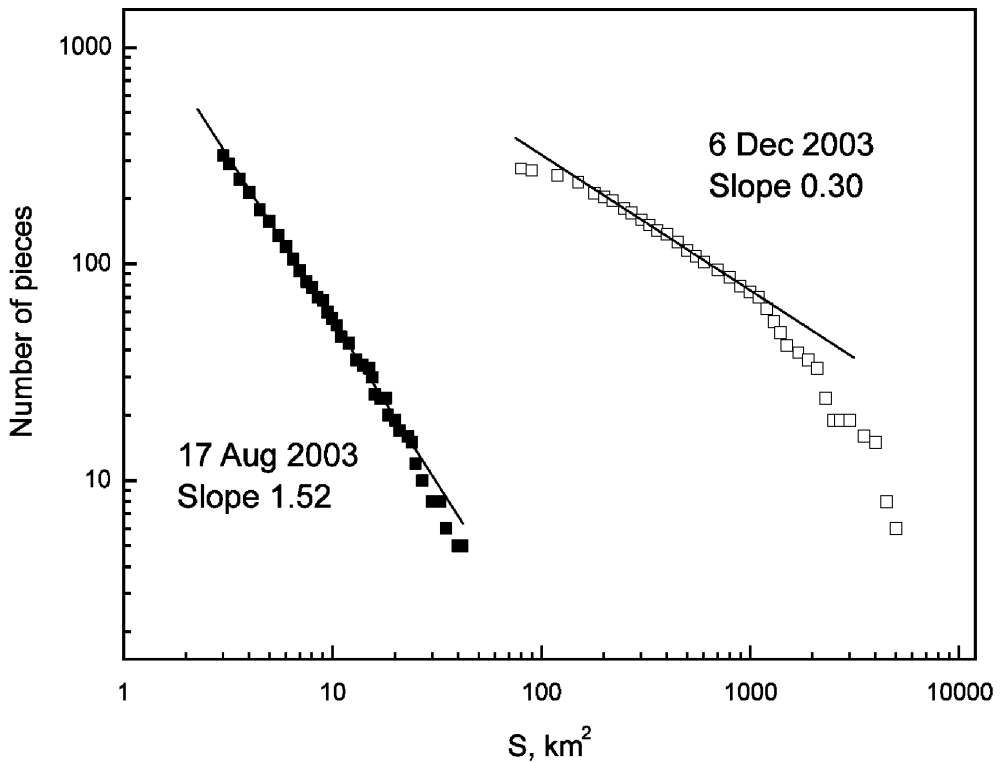

Fig. 2. Floe size distributions in the vicinity of the North Pole. From satellite images (not shown) taken on 17 August 2003 and on 6 December 2003.

\section{$4,1433-1448,2010$}

\section{Mechanical processes in Arctic sea ice}
A. Chmel et al.

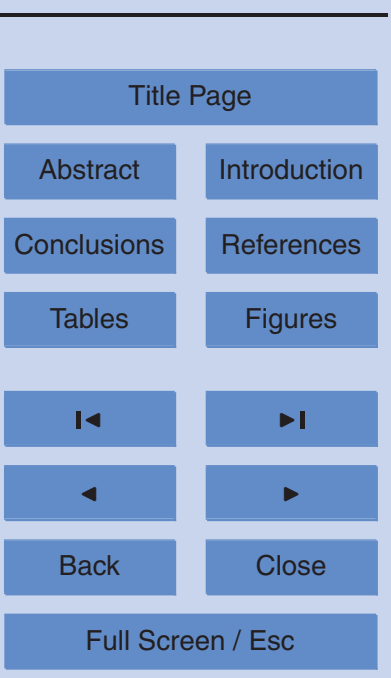

Printer-friendly Version

Interactive Discussion 


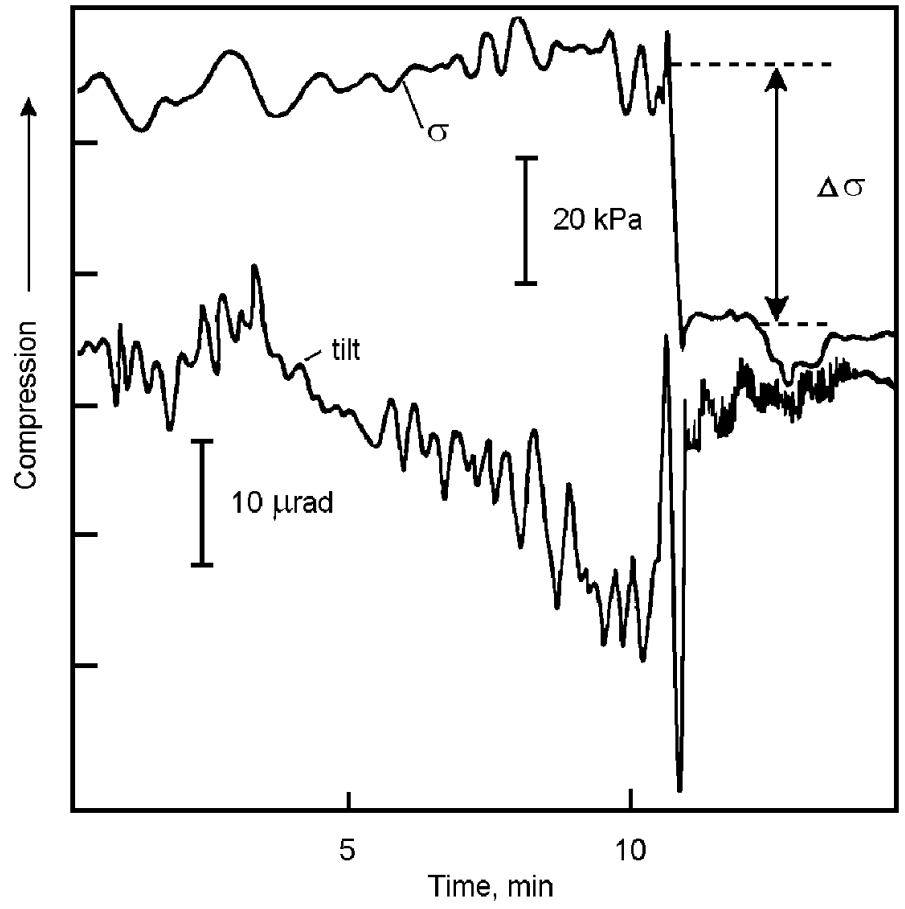

Fig. 3. Stress discharge in compressed sea ice as detected by tiltmeter (curve "tilt") and stress sensor (curve $\sigma$ ). From Smirnov (1996).
TCD

$4,1433-1448,2010$

\section{Mechanical} processes in Arctic sea ice
A. Chmel et al.

Title Page

Abstract

Introduction

Conclusions

References

Tables

Figures

14

4

Back

Close

\section{Full Screen / Esc}

Printer-friendly Version

Interactive Discussion

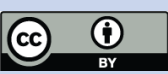




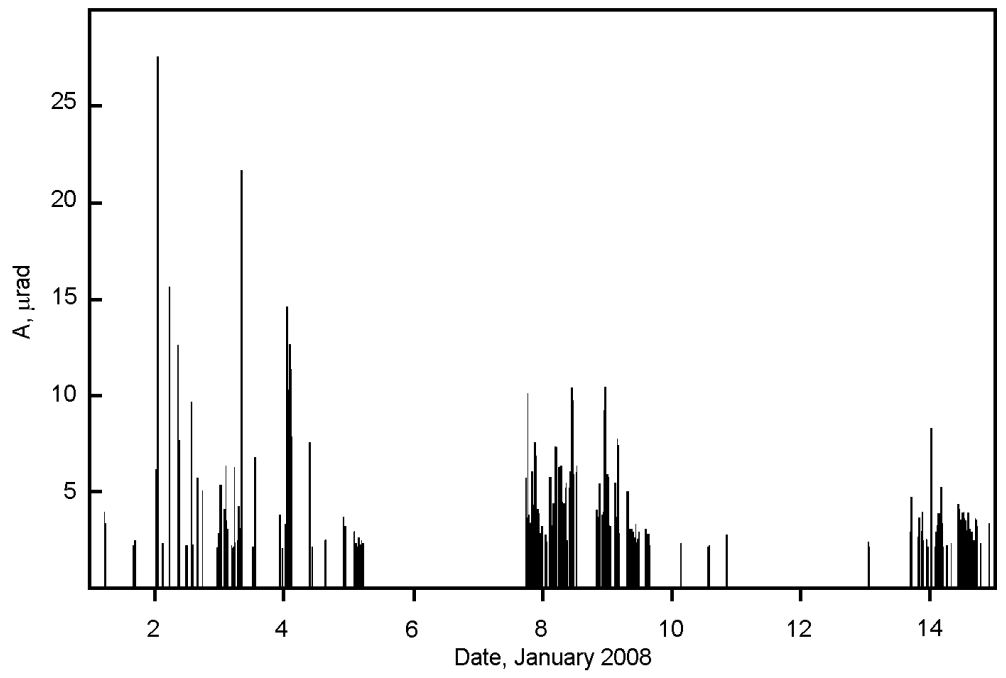

processes in Arctic sea ice

A. Chmel et al.

Title Page

Abstract

Introduction

Conclusions

References

Tables

Figures

14

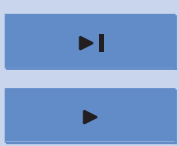

Back

Close

Full Screen / Esc

Printer-friendly Version

Interactive Discussion 


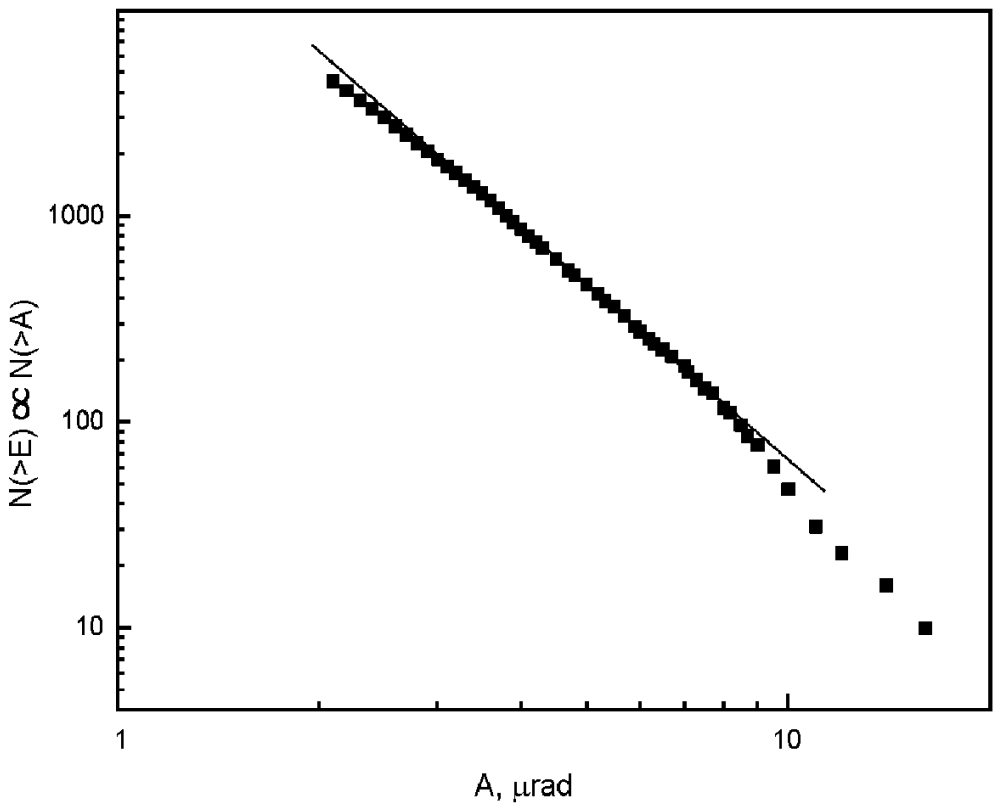

Fig. 5. Energy distribution in a sequence of elastic waves detected by tiltmeter in the frequency range of 0.5 to $5 \mathrm{~Hz}$ specific for mechanical processes related with ice fracturing. The straight line fits the power law (4).

\section{TCD}

$4,1433-1448,2010$

\section{Mechanical \\ processes in Arctic sea ice}

A. Chmel et al.

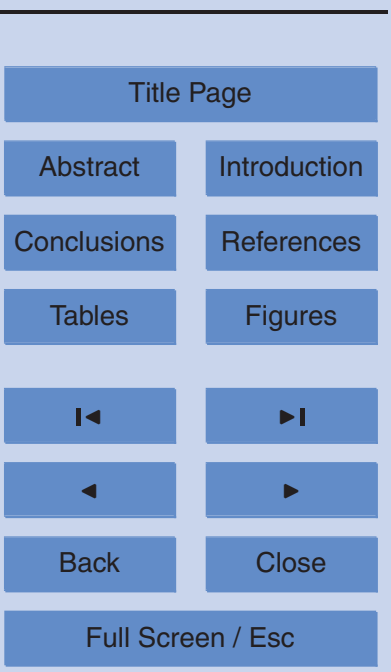

Printer-friendly Version

Interactive Discussion 


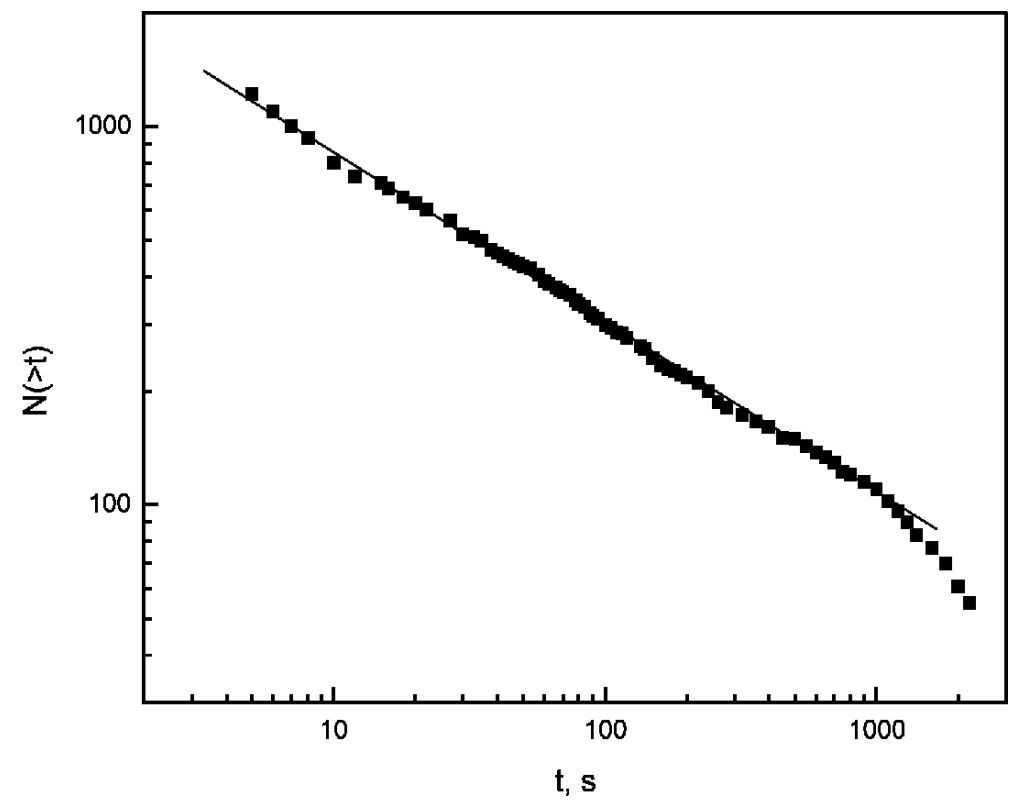

\section{TCD}

$4,1433-1448,2010$

\section{Mechanical processes in Arctic sea ice}

A. Chmel et al.

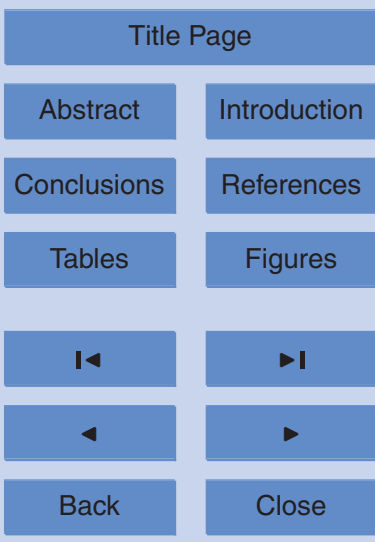

Fig. 6. Recurrence times distribution of tiltmeter signals shown in Fig. 4. The straight line fits the power law (5).

Full Screen / Esc

Printer-friendly Version

Interactive Discussion

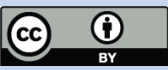

